\title{
CERTAIN NEW CLASSES OF MEROMORPHIC FUNCTIONS RELATED WITH A CONVOLUTION OPERATOR
}

\author{
SAQIB HUSSAIN, ADIL WAHID BUTT AND MUHAMMAD ZAHID
}

\begin{abstract}
Recently, Hussain [Hussain, Saqib (2010). Some applications of Miller-Mocanu lemma on certain classes of meromorphic functions, "Appl. Math. Comput.", 216(2010), 3016-3021], introduced certain new classes of meromorphic functions using newly defined convolution operators. The purpose of present investigation is to extend the work of Hussain by considering some new classes of meromorphic functions. We derive some inclusion relationships, coefficient bound, integral representation and extreme point theorem.
\end{abstract}

\section{Introduction}

Let $\Sigma$ denotes the class of meromorphic functions of the form

$$
f(z)=\frac{1}{z}+\sum_{k=0}^{\infty} a_{k} z^{k},
$$

which are analytic in the punctured open unit disc $D=\{z \in \mathbb{C}: 0<|z|<1\}$. Further, let $P_{k}(\gamma)$ be the class of functions $p(z)$ analytic in $E=D \cup\{0\}$ satisfying $p(0)=1$ and

$$
\int_{0}^{2 \pi}\left|\frac{\operatorname{Re} p(z)-\gamma}{1-\gamma}\right| d \theta \leq k \pi,
$$

where $z=r e^{i \theta}, k \geq 2,0 \leq \gamma<1$. This class was introduced by Padmanabhan and Parvatham [7]. For $\gamma=0$ we obtain the class $P_{k}$ defined by Pinchuk [9] and for $k=2, P_{2}(\gamma)=P(\gamma)$ is the class with real part greater than $\gamma$.

Also from (1.2) it can be seen that $p \in P_{k}(\gamma)$ if and only if

$$
p(z)=\left(\frac{k}{4}+\frac{1}{2}\right) p_{1}(z)-\left(\frac{k}{4}-\frac{1}{2}\right) p_{2}(z),
$$

Corresponding author: Saqib Hussain.

2010 Mathematics Subject Classification. 30C45, 30C50.

Key words and phrases. Meromorphic functions, convolution, integral operator. 
where $p_{1}, p_{2} \in P(\gamma)$ for $z \in E$. By $M C(\gamma)$ and $M S^{*}(\gamma)$, we mean the subclasses of meromorphic convex and meromorphic starlike functions of order $\gamma$ respectively. The class $\Sigma$ is closed under the convolution denoted and defined by

$$
(f * g)(z)=\frac{1}{z}+\sum_{k=0}^{\infty} a_{k} b_{k} z^{k},
$$

where

$$
f(z)=\frac{1}{z}+\sum_{k=0}^{\infty} a_{k} z^{k}, \quad g(z)=\frac{1}{z}+\sum_{k=0}^{\infty} b_{k} z^{k} .
$$

In [10] Yuan, et. all defined an operator $I_{n, \mu}: \Sigma \rightarrow \Sigma$ as follows:

$$
I_{n, \mu} f(z)=f_{n, \mu}(z) * f(z)
$$

where

$$
f_{n, \mu}(z) * \frac{1}{z(1-z)^{n+1}}=\frac{1}{z(1-z)^{\mu}}, n>-1, \mu>0, z \in D .
$$

From (1.3) and (1.4), it implies that

$$
I_{n, \mu} f(z)=\frac{1}{z}+\sum_{k=0}^{\infty} \frac{(\mu)_{k+1}}{(n+1)_{k+1}} a_{k} z^{k},
$$

where $(a)_{k}$ is the Pochhammer symbol defined as

$$
(a)_{0}=1, \quad(a)_{k}=a(a+1)(a+2) \cdots(a+k-1), k \in \mathbb{N} .
$$

From (1.5), it can be easily verified that

$$
z\left(I_{n+1, \mu} f(z)\right)^{\prime}=(n+1) I_{n, \mu} f(z)-(n+2) I_{n+1, \mu} f(z),
$$

and

$$
z\left(I_{n, \mu} f(z)\right)^{\prime}=\mu I_{n, \mu+1} f(z)-(\mu+1) I_{n, \mu} f(z) .
$$

The operator $I_{n, \mu}$ was extensively studied by several authors and obtained numerous results. For details, see [1-5,7] and [9]. Furthermore, for $c>0$ the generalized Bernardi operator is defined as

$$
J_{c} f(z)=\frac{c}{z^{c+1}} \int_{0}^{z} t^{c} f(t) d t
$$

From (1.7), we have

$$
(c+1) J_{c} f(z)+z\left(J_{c} f(z)\right)^{\prime}=c f(z), z \in D .
$$

Using the operator $I_{n, \mu}$, we define some new classes of meromorphic functions as follows:

Definition 1.1. Let $f(z) \in \Sigma, n>-1, \mu>0, \lambda \geq 0,0 \leq \gamma<1, z \in D$, then $f(z) \in M Q_{k}(n, \mu, \lambda, \gamma)$ if and only if

$$
-\left[\frac{z\left(I_{n, \mu} f(z)\right)^{\prime}+\lambda z^{2}\left(I_{n, \mu} f(z)\right)^{\prime \prime}}{(1-\lambda)\left(I_{n, \mu} f(z)\right)+\lambda z\left(I_{n, \mu} f(z)\right)^{\prime}}\right] \in P_{k}(\gamma) \text {. }
$$


Definition 1.2. Let $f(z) \in \Sigma, n>-1, \mu>0,0 \leq \gamma<1, z \in D$, then $f \in M R_{k}(n, \mu, \gamma)$ if and only if

$$
-\frac{z\left(I_{n, \mu} f(z)\right)^{\prime}}{I_{n, \mu} f(z)} \in P_{k}(\gamma)
$$

Definition 1.3. Let $f(z) \in \Sigma, n>-1, \mu>0, \lambda \geq 0,0 \leq \gamma<1,0 \leq \delta<1, m>0, z \in D$, then $f \in$ $M M_{k}(n, \mu, \lambda, \gamma, \delta)$ if and only if there exists $g \in M R_{2}(n, \mu, \delta)$ such that

$$
\left[(1-\lambda)\left[\frac{I_{n, \mu} f(z)}{I_{n, \mu} g(z)}\right]^{m}+\lambda\left[\frac{I_{n, \mu+1} f(z)}{I_{n, \mu+1} g(z)}\right]\left[\frac{I_{n, \mu} f(z)}{I_{n, \mu} g(z)}\right]^{m-1}\right] \in P_{k}(\gamma) .
$$

Remark 1. For special values of parameters $n, \mu, \gamma$ and $k$, we have many known classes of meromorphic functions, see [3, 8, 9] .

\section{Preliminaries Results}

Lemma 2.1 ([4]). Let $u=u_{1}+i u_{2}$ and $v=v_{1}+i v_{2}$ and let $\Psi(u, v)$ be a complex valued function satisfying the conditions:

(i) $\Psi(u, v)$ is continuous in $D \subset C^{2}$.

(ii) $(1,0) \in D$ and $\operatorname{Re} \Psi(1,0)>0$.

(iii) $\operatorname{Re} \Psi\left(i u_{2}, v_{1}\right) \leqslant 0$ whenever $\left(i u_{2}, v_{1}\right) \in D$ and $v_{1} \leqslant-\frac{1}{2}\left(1+u_{2}^{2}\right)$.

If $h(z)$ is a function analytic in $E$ such that $\left(h(z), z h^{\prime}(z) \in D\right.$ and

$\operatorname{Re} \Psi\left(h(z), z h^{\prime}(z)>0\right.$ for $z \in E$, then $\operatorname{Re} h(z)>0$ in $E$.

Note: Throughout in this article, we will use $n>-1, \mu>0, \lambda \geq 0,0 \leq \gamma<1,0 \leq \delta<1, m>0$ unless otherwise stated.

\section{Main Results}

Theorem 3.1.

$$
M Q_{k}(n, \mu+1, \lambda, \gamma) \subset M Q_{k}(n, \mu, \lambda, \beta),
$$

where

$$
\beta=\frac{1}{4}\left[(3+2 \mu+2 \gamma)-\sqrt{(3+2 \mu+2 \gamma)^{2}-8(2 \gamma+2 \mu \gamma+1)}\right]
$$

Proof. Let $f(z) \in M Q_{k}(n, \mu+1, \lambda, \gamma)$ and let

$$
-\left[\frac{z\left(I_{n, \mu} f(z)\right)^{\prime}+\lambda z^{2}\left(I_{n, \mu} f(z)\right)^{\prime \prime}}{(1-\lambda)\left(I_{n, \mu} f(z)\right)+\lambda z\left(I_{n, \mu} f(z)\right)^{\prime}}\right]=H(z),
$$


where $H(z)$ is analytic in $E$ and $H(0)=1$. Using (1.6) and (3.1) we obtain

$$
-\left[\frac{z\left(I_{n, \mu+1} f(z)\right)^{\prime}+\lambda z^{2}\left(I_{n, \mu+1} f(z)\right)^{\prime \prime}}{(1-\lambda)\left(I_{n, \mu+1} f(z)\right)+\lambda z\left(I_{n, \mu+1} f(z)\right)^{\prime}}\right]=H(z)+\frac{z H^{\prime}(z)}{-H(z)+(\mu+1)} \in P_{k}(\gamma) .
$$

Let

$$
\Phi_{\mu}(z)=\frac{1}{\mu+1}\left[\frac{1}{z}+\sum_{k=0}^{\infty} z^{k}\right]+\frac{\mu}{\mu+1}\left[\frac{1}{z}+\sum_{k=0}^{\infty} k z^{k}\right]
$$

then

$$
H(z) * z \Phi_{\mu}(z)=H(z)+\frac{z H^{\prime}(z)}{-H(z)+(\mu+1)} .
$$

Let

$$
H(z)=\left(\frac{k}{4}+\frac{1}{2}\right) h_{1}(z)-\left(\frac{k}{4}-\frac{1}{2}\right) h_{2}(z) .
$$

From (3.1), (3.3) and (3.4), we have

$$
\begin{aligned}
& -\left[\frac{z\left(I_{n, \mu+1} f(z)\right)^{\prime}+\lambda z^{2}\left(I_{n, \mu+1} f(z)\right)^{\prime \prime}}{(1-\lambda)\left(I_{n, \mu+1} f(z)\right)+\lambda z\left(I_{n, \mu+1} f(z)\right)^{\prime}}\right] \\
& \quad=\left(\frac{k}{4}+\frac{1}{2}\right)\left\{h_{1}(z)+\frac{z h_{1}^{\prime}(z)}{-h_{1}(z)+(\mu+1)}\right\}-\left(\frac{k}{4}-\frac{1}{2}\right)\left\{h_{2}(z)+\frac{z h_{2}^{\prime}(z)}{-h_{2}(z)+(\mu+1)}\right\} .
\end{aligned}
$$

Since $f(z) \in M Q_{k}(n, \mu+1, \lambda, \gamma)$, therefore

$$
h_{i}(z)+\frac{z h_{i}^{\prime}(z)}{-h_{i}(z)+(\mu+1)} \in P(\gamma) \text { for } i=1,2 ; z \in E .
$$

Let $h_{i}(z)=\beta+(1-\beta) p_{i}(z)$ for $i=1,2$. Then

$$
\left[[\beta-\gamma]+(1-\beta) p_{i}(z)+\frac{(1-\beta) z p_{i}^{\prime}(z)}{-(1-\beta) p_{i}(z)+(1+\mu-\beta)}\right] \in P \text { for } i=1,2 ; z \in E .
$$

We formulate a functional $\Psi(u, v)$ by taking $u=u_{1}+i u_{2}=p_{i}(z)$ and $v=v_{1}+i v_{2}=z p_{i}^{\prime}(z)$, then

$$
\Psi(u, v)=(\beta-\gamma)+(1-\beta) u+\frac{(1-\beta) v}{-(1-\beta) u+(1+\mu-\beta)} .
$$

The first two conditions of Lemma 2.1 are obviously satisfied for $\Psi(u, v)$. For the third condition, we proceed as follows:

$$
\operatorname{Re} \Psi\left(i u_{2}, v_{1}\right)=(\beta-\gamma)+\frac{(1-\beta)(1+\mu-\beta) v_{1}}{(1+\mu-\beta)^{2}+(1-\beta)^{2} u_{2}^{2}} .
$$

By putting $v_{1} \leq-\frac{1}{2}\left(1+u_{2}^{2}\right)$, we have

$$
\operatorname{Re} \Psi\left(i u_{2}, v_{1}\right) \leq \frac{A+B u_{2}^{2}}{2 C}
$$


where

$$
\begin{aligned}
& A=2(\beta-\gamma)(1+\mu-\beta)^{2}-(1-\beta)(1+\mu-\beta), \\
& B=2(\beta-\gamma)(1-\beta)^{2}-(1-\beta)(1+\mu-\beta), \\
& C=(1+\mu-\beta)^{2}+(1-\beta)^{2} u_{2}^{2} .
\end{aligned}
$$

We note that $\operatorname{Re} \Psi\left(i u_{2}, v_{1}\right) \leq 0$ if $A \leq 0$ and $B \leq 0$.From $A \leq 0$, we obtain

$$
\beta=\frac{1}{4}\left[(3+2 \mu+2 \gamma)-\sqrt{(3+2 \mu+2 \gamma)^{2}-8(2 \gamma+2 \mu \gamma+1)}\right] .
$$

By virtue of Lemma 2.1, we see that $p_{i} \in P$, for $i=1,2$ and $z \in E$. Hence $h_{i}(z) \in P(\beta)$ which implies $H(z) \in P_{k}(\beta)$ and consequently $f(z) \in M Q_{k}(n, \mu, \lambda, \beta)$.

Next we show that the class $M Q_{k}(n, \mu, \lambda, \gamma)$ is closed under the Bernardi integral operator.

Theorem 3.2. Let $f(z) \in \Sigma$. If $f(z) \in M Q_{k}(n, \mu, \lambda, \gamma)$ then $J_{c} f(z) \in M Q_{k}(n, \mu, \lambda, \gamma)$.

Proof. Let $f(z) \in M Q_{k}(n, \mu, \lambda, \gamma)$ and set

$$
-\left[\frac{z J_{c}\left(I_{n, \mu} f(z)\right)^{\prime}+\lambda z^{2} J_{c}\left(I_{n, \mu} f(z)\right)^{\prime \prime}}{(1-\lambda) J_{c}\left(I_{n, \mu} f(z)\right)+\lambda z J_{c}\left(I_{n, \mu} f(z)\right)^{\prime}}\right]=H(z),
$$

where $H(z)$ is analytic in $E$ and $H(0)=1$. Using (1.8) and (3.4) we get

$$
-\left[\frac{z J_{c}\left(I_{n, \mu+1} f(z)\right)^{\prime}+\lambda z^{2} J_{c}\left(I_{n, \mu+1} f(z)\right)^{\prime \prime}}{(1-\lambda) J_{c}\left(I_{n, \mu+1} f(z)\right)+\lambda z J_{c}\left(I_{n, \mu+1} f(z)\right)^{\prime}}\right]=H(z)+\frac{z H^{\prime}(z)}{-H(z)+(c+1)} \in P_{k}(\gamma)
$$

Now using the same steps as in Theorem 3.1, we can prove that $J_{c} f(z) \in M Q_{k}(n, \mu, \lambda, \gamma)$, which completes the proof.

Theorem 3.3. The necessary and sufficient condition for $f(z)$ defined by (1.1) to be in the class $M Q_{2}(n, \mu, \lambda, \gamma)$ is

$$
\sum_{k=0}^{\infty}\left[k^{2}+\gamma(1+k)\right] \frac{(\mu)_{k+1}}{(n+1)_{k+1}}\left|a_{k}\right| \leq(\gamma-1)(2 \lambda-1) .
$$

Proof. Let $f(z) \in M Q_{2}(n, \mu, \lambda, \gamma)$, then

$$
\operatorname{Re}\left[\frac{z\left(I_{n, \mu} f(z)\right)^{\prime}+\lambda z^{2}\left(I_{n, \mu} f(z)\right)^{\prime \prime}}{(1-\lambda)\left(I_{n, \mu} f(z)\right)+\lambda z\left(I_{n, \mu} f(z)\right)^{\prime}}\right]<-\gamma,
$$

which implies using (1.5)

$$
\operatorname{Re}\left[\frac{\frac{1}{z}(2 \lambda-1)+\sum_{k=0}^{\infty} k^{2} \frac{(\mu)_{k+1}}{(n+1)_{k+1}} a_{k} z^{k}}{-\frac{1}{z}(2 \lambda-1)+\sum_{k=0}^{\infty}(1+k) \frac{(\mu)_{k+1}}{(n+1)_{k+1}} a_{k} z^{k}}\right]<-\gamma .
$$


Letting $z \rightarrow 1^{-}$along the real axis, we have

$$
\left[\frac{(2 \lambda-1)+\sum_{k=0}^{\infty} k^{2} \frac{(\mu)_{k+1}}{(n+1)_{k+1}} a_{k}}{-(2 \lambda-1)+\sum_{k=0}^{\infty}(1+k) \frac{(\mu)_{k+1}}{(n+1)_{k+1}} a_{k}}\right]+\gamma<0
$$

By maximum modulus principle, the above equation leads to the desired inequality

$$
\sum_{k=0}^{\infty}\left[k^{2}+\gamma(1+k)\right] \frac{(\mu)_{k+1}}{(n+1)_{k+1}}\left|a_{k}\right| \leq(\gamma-1)(2 \lambda-1) .
$$

Conversely, suppose (3.8) is true for $z \in D$. Then

$$
\operatorname{Re}\left[\frac{z\left(I_{n, \mu} f(z)\right)^{\prime}+\lambda z^{2}\left(I_{n, \mu} f(z)\right)^{\prime \prime}}{(1-\lambda)\left(I_{n, \mu} f(z)\right)+\lambda z\left(I_{n, \mu} f(z)\right)^{\prime}}\right]+\gamma<0,
$$

if

$$
\operatorname{Re}\left[\frac{\frac{1}{z}(2 \lambda-1)+\sum_{k=0}^{\infty} k^{2} \frac{(\mu)_{k+1}}{(n+1)_{k+1}} a_{k} z^{k}}{-\frac{1}{z}(2 \lambda-1)+\sum_{k=0}^{\infty}(1+k) \frac{(\mu)_{k+1}}{(n+1)_{k+1}} a_{k} z^{k}}\right]+\gamma<0,
$$

that is if

$$
\sum_{k=0}^{\infty}\left[k^{2}+\gamma(1+k)\right] \frac{(\mu)_{k+1}}{(n+1)_{k+1}}\left|a_{k}\right| \leq(\gamma-1)(2 \lambda-1),
$$

which completes the proof.

Corollary 3.1. Let the function $f(z)$ defined by (1.1) belongs to the class $M Q_{2}(n, \mu, \lambda, \gamma)$. Then

$$
\left|a_{k}\right| \leq \frac{(\gamma-1)(2 \lambda-1)}{\left[k^{2}+\gamma(1+k)\right]} \frac{(n+1)_{k+1}}{(\mu)_{k+1}} .
$$

This inequality is sharp and the extremal function is given by

$$
f(z)=\frac{1}{z}+\frac{(\gamma-1)(2 \lambda-1)}{\left[k^{2}+\gamma(1+k)\right]} \frac{(n+1)_{k+1}}{(\mu)_{k+1}} z^{k} .
$$

Theorem 3.4. Let the functions $f_{j}(z) \in M Q_{2}(n, \mu, \lambda, \gamma)$ be defined as

$$
f_{0}(z)=\frac{1}{z} \text { and } f_{n}(z)=\frac{1}{z}+\frac{(\gamma-1)(2 \lambda-1)}{\left[k^{2}+\gamma(1+k)\right]} \frac{(n+1)_{k+1}}{(\mu)_{k+1}} z^{k} \text { for } n \in \mathbb{N} \text {. }
$$

Then $f(z) \in M Q_{2}(n, \mu, \lambda, \gamma)$ if and only if $f(z)$ can be expressed in the form

$$
f(z)=\sum_{k=0}^{\infty} \xi_{k} f_{k}(z) \text { where } \xi_{k} \geq 0 \text { and } \sum_{k=0}^{\infty} \xi_{k}=1 .
$$


Proof. Suppose that $f(z)$ can be expressed in the form (3.9), then

$$
f(z)=\frac{1}{z}+\sum_{k=1}^{\infty} \frac{(\gamma-1)(2 \lambda-1)}{\left[k^{2}+\gamma(1+k)\right]} \xi_{k} z^{k}
$$

Consider

$$
\sum_{k=0}^{\infty}\left[\left[k^{2}+\gamma(1+k)\right] \frac{(\mu)_{k+1}}{(n+1)_{k+1}}\right] \xi_{k} \frac{(\gamma-1)(2 \lambda-1)}{\left[k^{2}+\gamma(1+k)\right]} \frac{(n+1)_{k+1}}{(\mu)_{k+1}} \leq(\gamma-1)(2 \lambda-1),
$$

so by (3.8), we have $f(z) \in M Q_{2}(n, \mu, \lambda, \gamma)$. Conversely let $f(z) \in M Q_{2}(n, \mu, \lambda, \gamma)$, and set

$$
\xi_{k}=\frac{\left[k^{2}+\gamma(1+k)\right]}{(\gamma-1)(2 \lambda-1)} \frac{(\mu)_{k+1}}{(n+1)_{k+1}}\left|a_{k}\right|, k=1,2, \ldots
$$

with $\xi_{0}=1-\sum_{k=1}^{\infty} \xi_{k}$. We obtain

$$
\sum_{k=1}^{\infty} \xi_{k}=\sum_{k=1}^{\infty} \frac{\left[k^{2}+\gamma(1+k)\right]}{(\gamma-1)(2 \lambda-1)} \frac{(\mu)_{k+1}}{(n+1)_{k+1}}\left|a_{k}\right| \leq 1,
$$

then $f(z)=\sum_{k=0}^{\infty} \xi_{k} f_{k}(z)$ where $\xi_{k} \geq 0$ and $\sum_{k=0}^{\infty} \xi_{k}=1$. This completes the proof.

Theorem 3.5. Let $f(z) \in M M_{k}(n, \mu, \lambda, \gamma, \delta)$ w.r.t $g(z) \in M R_{2}(n, \mu, \gamma)$, then $\left[\frac{I_{n, \mu} f(z)}{I_{n, \mu} g(z)}\right]^{m} \in P_{k}(\beta)$, where

$$
\beta=\frac{q_{1}+2 \gamma}{q_{1}+2} \text { and } q_{1}(z)=\frac{\lambda}{\mu m} \operatorname{Re}\left(\frac{I_{n, \mu} g(z)}{I_{n, \mu+1} g(z)}\right) .
$$

Proof. Let $f(z) \in M M_{k}(n, \mu, \lambda, \gamma, \delta)$ and let

$$
\left[\frac{I_{n, \mu} f(z)}{I_{n, \mu} g(z)}\right]^{m}=H(z)=\left(\frac{k}{4}+\frac{1}{2}\right) h_{1}(z)-\left(\frac{k}{4}-\frac{1}{2}\right) h_{2}(z) .
$$

It can be easily verified that

$$
\begin{aligned}
& {\left[(1-\lambda)\left[\frac{I_{n, \mu} f(z)}{I_{n, \mu} g(z)}\right]^{m}+\lambda\left[\frac{I_{n, \mu+1} f(z)}{I_{n, \mu+1} g(z)}\right]\left[\frac{I_{n, \mu} f(z)}{I_{n, \mu} g(z)}\right]^{m-1}\right]} \\
& =H(z)+\frac{\lambda z H^{\prime}(z) Q(z)}{\mu m} \in P_{k}(\gamma),
\end{aligned}
$$

where $Q(z)=\frac{I_{n, \mu} g(z)}{I_{n, \mu+1} g(z)}$. Let $q(z)=\frac{\lambda Q(z)}{\mu m}$, then by (3.10) and (3.11)

$$
\begin{aligned}
& {\left[(1-\lambda)\left[\frac{I_{n, \mu} f(z)}{I_{n, \mu} g(z)}\right]^{m}+\lambda\left[\frac{I_{n, \mu+1} f(z)}{I_{n, \mu+1} g(z)}\right]\left[\frac{I_{n, \mu} f(z)}{I_{n, \mu} g(z)}\right]^{m-1}\right]} \\
& \quad=\left(\frac{k}{4}+\frac{1}{2}\right)\left[h_{1}(z)+z h_{1}^{\prime}(z) q(z)\right]-\left(\frac{k}{4}-\frac{1}{2}\right)\left[h_{2}(z)+z h_{2}^{\prime}(z) q(z)\right] .
\end{aligned}
$$


Since $f(z) \in M M_{k}(n, \mu, \lambda, \gamma, \delta)$, so by (3.12),

$$
h_{i}(z)+z h_{i}^{\prime}(z) q(z) \in P(\gamma) \text { for } i=1,2 ; z \in E
$$

Let $h_{i}(z)=\beta+(1-\beta) p_{i}(z)$ for $i=1,2$. Then

$$
\left[(\beta-\gamma)+(1-\beta) p_{i}(z)+(1-\beta) z p_{i}^{\prime}(z) q(z)\right] \in P \text { for } i=1,2 ; z \in E
$$

We formulate a functional $\Psi(u, v)$ by taking $u=u_{1}+i u_{2}=p_{i}(z)$ and $v=v_{1}+i v_{2}=z p_{i}^{\prime}(z)$, then

$$
\Psi(u, v)=(\beta-\gamma)+(1-\beta) u+(1-\beta) v\left[q_{1}+i q_{2}\right]
$$

The first two conditions of Lemma 2.1 are obviously satisfied for $\Psi(u, v)$. For the third condition, we proceed as follows:

$$
\operatorname{Re} \Psi\left(i u_{2}, \nu_{1}\right)=(\beta-\gamma)+(1-\beta) \nu_{1} q_{1}
$$

By putting $v_{1} \leq-\frac{1}{2}\left(1+u_{2}^{2}\right)$, we have

$$
\operatorname{Re} \Psi\left(i u_{2}, v_{1}\right) \leq A+B u_{2}^{2},
$$

where

$$
\begin{aligned}
A & =(\beta-\gamma)-\frac{1}{2}(1-\beta) q_{1}, \\
B & =-\frac{1}{2}(1-\beta) q_{1} \leq 0
\end{aligned}
$$

We note that $\operatorname{Re} \Psi\left(i u_{2}, v_{1}\right) \leq 0$ if $A \leq 0$. From $A \leq 0$, we obtain

$$
\beta=\frac{q_{1}+2 \gamma}{q_{1}+2}
$$

Using Lemma 2.1, we see that $p_{i} \in P$, for $i=1,2$ and $z \in E$. Hence $h_{i}(z) \in P(\beta)$ which implies $H(z) \in P_{k}(\beta)$. Our proof is complete.

Theorem 3.6. For $0 \leq \lambda_{1}<\lambda_{2}$,

$$
M M_{k}\left(n, \mu, \lambda_{2}, \gamma, \delta\right) \subset M M_{k}\left(n, \mu, \lambda_{1}, \gamma, \delta\right)
$$

Proof. Let $f(z) \in M M_{k}\left(n, \mu, \lambda_{2}, \gamma, \delta\right)$, then

$$
\left[\left(1-\lambda_{2}\right)\left[\frac{I_{n, \mu} f(z)}{I_{n, \mu} g(z)}\right]^{m}+\lambda_{2}\left[\frac{I_{n, \mu+1} f(z)}{I_{n, \mu+1} g(z)}\right]\left[\frac{I_{n, \mu} f(z)}{I_{n, \mu} g(z)}\right]^{m-1}\right]=H_{2}(z) \in P_{k}(\gamma)
$$


by Theorem ??,

$$
\left[\frac{I_{n, \mu} f(z)}{I_{n, \mu} g(z)}\right]^{m}=H_{1}(z) \in P_{k}(\beta) \subset P_{k}(\gamma) .
$$

If $\lambda_{1}=0$, the proof is immediate from Theorem ??. Therefore we consider $\lambda_{1}>0$,

$$
\left[\left(1-\lambda_{1}\right)\left[\frac{I_{n, \mu} f}{I_{n, \mu} g}\right]^{m}+\lambda_{1}\left[\frac{I_{n, \mu+1} f}{I_{n, \mu+1} g}\right]\left[\frac{I_{n, \mu} f}{I_{n, \mu} g}\right]^{m-1}\right]=\left(1-\frac{\lambda_{1}}{\lambda_{2}}\right) H_{1}(z)+\frac{\lambda_{1}}{\lambda_{2}} H_{2}(z) .
$$

Since $P_{k}(\gamma)$ is a convex set [5], thus right side of (3.13) belongs to $P_{k}(\gamma)$. Thus $f(z) \in M M_{k}$ $\left(n, \mu, \lambda_{1}, \gamma, \delta\right)$.

Theorem 3.7. Let $f(z) \in M R_{2}(n, \mu, \gamma)$, then $J_{c} f(z) \in M R_{2}(n, \mu, \gamma)$.

Proof. Let $f(z) \in M R_{2}(n, \mu, \gamma)$ and set

$$
-\frac{z J_{c}\left(I_{n, \mu} f(z)\right)^{\prime}}{J_{c}\left(I_{n, \mu} f(z)\right)}=H(z)
$$

(3.14) together with (1.8) implies that

$$
-\frac{z\left(I_{n, \mu} f(z)\right)^{\prime}}{\left(I_{n, \mu} f(z)\right)}=H(z)+\frac{z H^{\prime}(z)}{-H(z)+(c+1)} .
$$

Now following the same steps as in Theorem 3.1, we obtain the required result.

\section{Acknowledgement}

The authors would like to thank teh refrees for their constructive suggestions and Dr. khan Gul Jadoon, Director CIIT Abbottabad for providing excellent research facilities.

\section{References}

[1] N. E. Cho and K. I. Noor, Inclusion properties for certain classes of meromorphic functions associated with Choi-Saigo-Srivastava operator, J. Math. Anal. Appl., 320(2006), 779-786.

[2] J. H. Choi, M. Saigo and H. M. Srivastava, Some inclusion properties of a certain family of integral operators, J. Math. Anal. Appl., 276(2002), 432-445.

[3] S. Hussain, Some applications of Miller-Mocanu lemma on certain classes of meromorphic functions, Appl. Math. Comput., 216(2010), 3016-3021.

[4] S. S. Miller, Differential inequalities and Caratheodory functions, Bull. Amer. Math. Soc., 81 (1975), 79-81.

[5] K. I. Noor, On subclasses of close-to-convex functions of higher order, Int. J. Math. Math. Sci., 15(1992), 279290.

[6] K. I. Noor, On certain classes of meromorphic functions involving integral operators, J. Ineq. Pure. Appl. Math, 7(2006), 1-8.

[7] K. S. Padmanabhan and R. Parvatham, Properties of a class of functions with bounded boundary rotation, Ann. Polon. Math., 31(1975), 311-323. 
[8] K. Piejko and J. Sokół, Subclasses of meromorphic functions associated with the Cho-Kwon-Srivastava operator, J. Math. Anal. Appl., 337(2008), 1261-1266.

[9] B. Pinchuk, Functions of bounded boundary rotation, Isr. J. Math., 10(1971), 6-16.

[10] S. M. Yuan, Z. M. Liu and H. M. Srivastava, Some inclusion relationships and integral-preserving properties of certain subclasses of meromorphic functions associated with a family of integral operators, J. Math. Anal. Appl., 337(2008), 505-515.

Department of Mathematics, COMSATS Institute of Information Technology, Abbottabad, Pakistan.

E-mail: saqib_math@yahoo.com

Department of Mathematics, COMSATS Institute of Information Technology, Abbottabad, Pakistan.

E-mail: adil.maths@yahoo.com

Department of Mathematics, COMSATS Institute of Information Technology, Abbottabad, Pakistan.

E-mail: zahid315@hotmail.com 